\title{
Application of pomelo essential oil (Citrus Grandis L.) in effective scenting of diffused products
}

\author{
Tran Thi Kim Ngan ${ }^{1,2}$, Thien Hien Tran ${ }^{1,2}$, Ly Thi Ngoc Minh ${ }^{3}$, Huynh Bao Long ${ }^{4}$, and Xuan \\ Tien $\mathrm{Le}^{5,6, *}$ \\ ${ }^{1}$ Institute of Environmental Sciences, Nguyen Tat Thanh University, Ho Chi Minh City, Vietnam \\ ${ }^{2}$ Faculty of Food and Environmental Engineering, Nguyen Tat Thanh University, Ho Chi Minh City, \\ Viet Nam \\ ${ }^{3}$ Branch of Green Power Co., LTD, Ben Tre Province, Vietnam \\ ${ }^{4} \mathrm{Ho}$ Chi Minh City University of Food Industry, Vietnam \\ ${ }^{5}$ Faculty of Chemical Engineering, Ho Chi Minh City University of Technology, Ho Chi Minh City, \\ Vietnam \\ ${ }^{6}$ Vietnam National University Ho Chi Minh City, Ho Chi Minh City, Vietnam
}

\begin{abstract}
In order to minimize the toxic effects of scent compounds from chemicals to the environment and humans. Besides, the increasing demand of consumers for natural compounds, so the research team has conducted a search for compounds with good odor-producing ability and high activity extracted from the species plants in nature. The study was carried out based on the investigation of influencing parameters in product formulations such as carrier, essential oil, emulsifier, solvent. Research results are based on the evaluation of the product's volatility and appearance. At the same time, it is also shown that pomelo essential oil has the ability to combine with many other ingredients in the product without being denatured, with the content of $4 \%$ pomelo essential oil is diffused with a pleasant scent that is not pungent and can High deodorizing capacity in the air. The obtained research results have opened new perspectives on the applicability and potential of essential oils in the aromatherapy industry or cosmetic field, used as an alternative to artificial fragrances with properties of environmental friendliness.
\end{abstract}

\section{Introduction}

Essential oils as a source of aromatherapy derived from natural plants are getting more and more attention and popularity. Studies aimed at quoting essential oils, determining physicochemical indices, and testing antimicrobial activity against pathogenic bacteria have increased in recent years [1-5]. Vietnam has favorable tropical and natural conditions for the formation and development of plant species, in which the essential oil-containing plants are rich and diverse [6-11]. Besides, the Citrus variety of the Rutaceae family with a pleasant aroma, high limonene content has great functions in food and cosmetic applications. Green-

* Corresponding author: tien.le@hcmut.edu.vn 
skinned pomelo (Citrus maxima (Burm.Merr.) or Citrus grandis (Osb.) - belonging to the family Rutaceae) is a popular fruit tree grown in the Mekong Delta in which many provinces such as Ben Tre are concentrated. Utilizing the source of pomelo peels (young pomelos fall during development, pomelo peels after production and processing) will help to obtain an abundant, stable, and cheap source of local raw materials for the process of producing essential oils and some products from pomelo peel and thereby increasing the value of pomelo trees. This is a source of raw materials for the production of essential oils that are stable in price and quality. Besides, the diversification of products based on pomelo essential oil will help create products of high economic value.

Pomelo peel contains a large amount of essential oil about $0.4-1 \%$ of the weight of the peel, this essential oil contains a mixture of volatile aromatics and has high economic value $[12,13]$. Pomelo essential oil usually contains limonene $(80-88 \%), \beta$-Pinene $(0.8-1.2 \%)$, linalool $(1.1 .-0.7 \%), \alpha$-terpinene $(0.7-1.0 \%)[13,14,15,16]$. According to studies, pomelo essential oil has many antibacterial activities against Staphylococcus aureus, Enterococcus faecalis, Staphylococcus epidermidis, Escherichia coli, Salmonella typhimurium, and Proteus vulgaris [17,18].

Due to their volatile and aromatic components, essential oils have various applications such as aromatherapy and perfumery. The health, beauty and relaxation benefits of essential oils make this natural product so popular. With the main effect of diffusing the aroma of essential oils to the outside, creating a fresh, clean space of bacteria and germs, they not only bring a pleasant scent but also help reduce stress extremely effectively. The creation of an essential oil diffuser has demonstrated an increased diffusion capacity and the effect of breaking down the essence of essential oils into hundreds of millions of smaller molecules so that they can quickly disperse into the environment helps create a gentle fragrance from nature. In addition, the use of essential oils as aromatherapy will minimize the use of aromatherapy from chemicals that can affect human health. On the other hand, in order to reduce the cost and reduce the essential oil content when used, a mixture of diffusion solution from pomelo essential oil was studied. The presence and combination of solubilizers, emulsifiers, diffusion aids, and proportions of pomelo essential oil have resulted in an environmental friendly diffusion solution, cost-effective and user-friendly. solve the surplus of pomelo peel by-products, improve the value of available pomelos in Vietnam. Therefore, the purpose of this study is to create diffused products from pomelo essential oil in order to use the most comprehensive and effective use of locally available raw materials to create products serving consumer needs use.

\section{Materials and methods}

\subsection{Raw materials and chemicals}

Pomelo material was collected from Ben Tre province, Vietnam. After importing, it is preliminarily processed, removing unsatisfactory components. Then the raw materials are washed to clean the sand, dirt, and microorganisms attached to the outside. Pomelo is peeled with about $0.5 \mathrm{~cm}$ thick. Pomelo peel was chopped to a size suitable for production requirements as in the study $\left(0.5 \times 0.5 \mathrm{~cm}^{2}\right)$. The materials are weighted and distillated with the appropriate amount of water required by each experiment. The obtained essential oil will be anhydrous with $\mathrm{Na}_{2} \mathrm{SO}_{4}$ and stored in the refrigerator. 


\subsection{Product blend process}

First, pomelo essential oil was dissolved with an emulsifier and the solvent was dissolved with a carrier oil which was gently stirred in 2 separate beakers to form a homogeneous mixture for $30 \mathrm{~min}$. After that, the mixture of cup solution 1 was added to beaker 2 and continued to stir until homogenized at room temperature and for 1 hour. Different fragrances will be added to the mixture to increase the diversity of the product. Eventually, we will get a diffused solution.

\subsection{Methods for sensory evaluation and volatility of products}

State of the product is to assess product homogeneity and clarity, the diffusion solution mixtures shall be contained in transparent vials and observed visually against a white background under the light. In addition, to evaluate the impact of the environment on the denaturation of the product, experiments were conducted to store the product in different conditions (room temperature and at $45^{\circ} \mathrm{C}$ ). Observe the following properties, which are state, color, smell, etc. Product volatility based on the mass of the product after evaporation compared to the original product used. Add $2 \mathrm{mg}$ of diffusion solution onto a petri dish, observe the evaporation time of the samples to be evaluated, when allowed to stabilize for a period of 1 hour, 2 hours, and 3 hours. Then, record the evaporated mass of the product.

\section{Results and discussion}

\subsection{The effect of essential oil contents on diffusion solutions}

The results of the evaluation of the evaporation capacity of the diffused product are shown in Figure 1. The analysis shows that the content of pomelo essential oil is proportional to the clarity of the product, besides the use of pomelo essential oil with too high a concentration also affects the taste of the product when it causes too strong a smell when used. Pomelo essential oil has many attractive aromatic compounds (myrcene and limonene) and some compounds with strong deodorizing and antibacterial properties ( $\alpha$-pinene and $\beta$-pinene) should be used as the main ingredients in the diffusion solution. The volatility of the solution increases with the essential oil content from $1-4 \%$ after 1 hour and 2 hours and 3 hours, when increasing the concentration of $5 \%$, the volatility tends to decrease slightly compared to that of the solution sample $4 \%$. On the other hand, when the essential oil content is high, the scent of the product will be dark. Therefore, the content of pomelo essential oil used at $4 \%$ is suitable for diffusion products. 


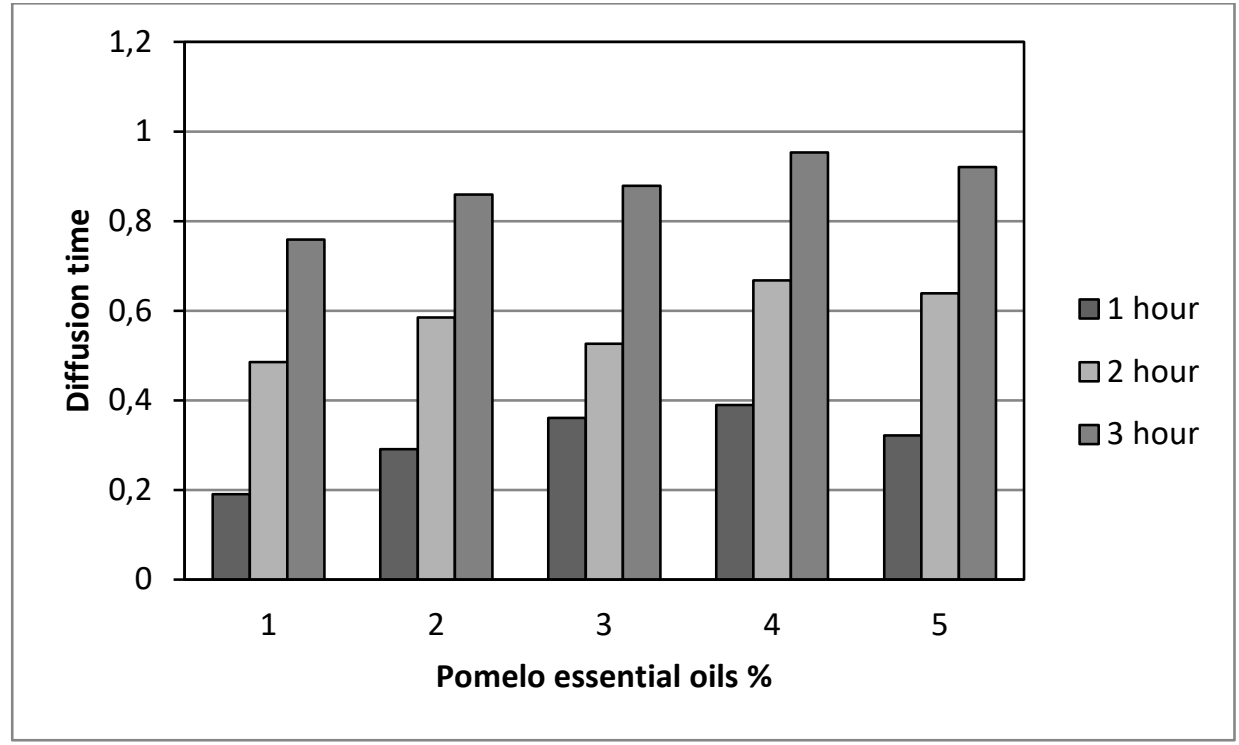

Fig. 1. The influence of pomelo essential oil content

\subsection{The effect of carrier substances contents on diffusion solutions}

The influence of carrier substances on the base of the diffused solution product is shown in Figure 2. The results of visual evaluation of the product when using a carrier type of Dipropylene glycol gives the best results from clarity, homogeneity, scent, $\mathrm{pH}$ of the solution. For coconut oil, the solution is cloudy and appears to have a clear separation and the oil layer will float to the top. For the carrier substances type experiment, when the time increased from 1-3 hours, the product diffusivity increased for Dipropylene glycol and Polythylene glycol.

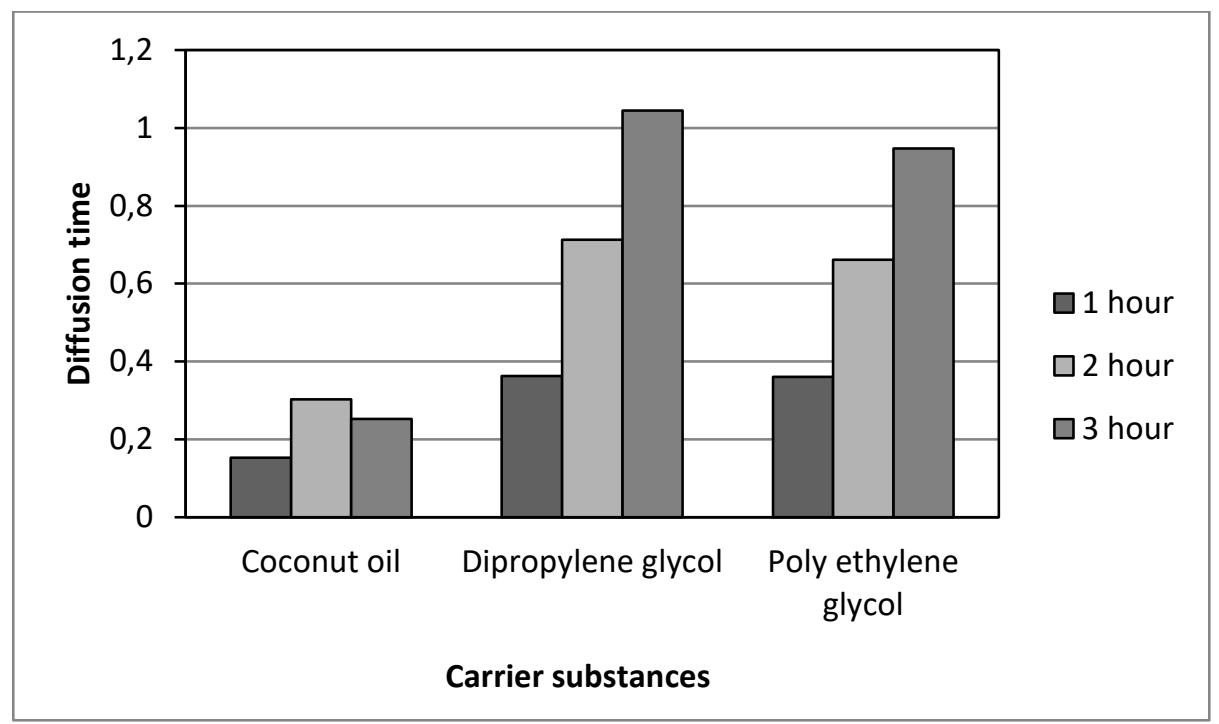

Fig. 2. The influence of the carrier substances on the base of the diffusion solution. 
In particular, the diffusion rate of the product at the 3 hour time period increased significantly compared to 2 hour and 1 hour for the Dipropylene glycol sample. Dipropylene glycol is a colorless, nearly odorless liquid with a high boiling point and low toxicity. Dipropylene glycol helps to dissolve and evenly disperse all ingredients in the formula without chemically altering the other ingredients. On the other hand, in terms of the product cost, Dipropylene glycol is cheaper than Polyethylene glycol despite similar properties. Therefore, use the Dipropylene glycol sample for the follow-up investigation.

The appearance of the product when using different solvent and carrier ratios, the results shows that with the ratio of ethanol: carrier, the diffusion solution is transparent. Diffusion solution using ethanol solvent has a pleasant aroma when using the ratio 5:5 and 6:4, the diffused solution has a stronger odor when increasing the ethanol ratio. The graph results show that the evaporation of the diffusion solution using the ratio of ethanol solvent and $6: 4$ carrier gives a much better evaporation efficiency than the other ratio. After 2 hours and 3 hours, the volatility of ethanol: carrier is $0.962-1.247$. The use of high ethanol content will cause the diffused product to have a strong odor that causes discomfort when used. Therefore, a 6:4 ethanol: carrier ratio is used as the solvent: carrier ratio for the diffusion product.

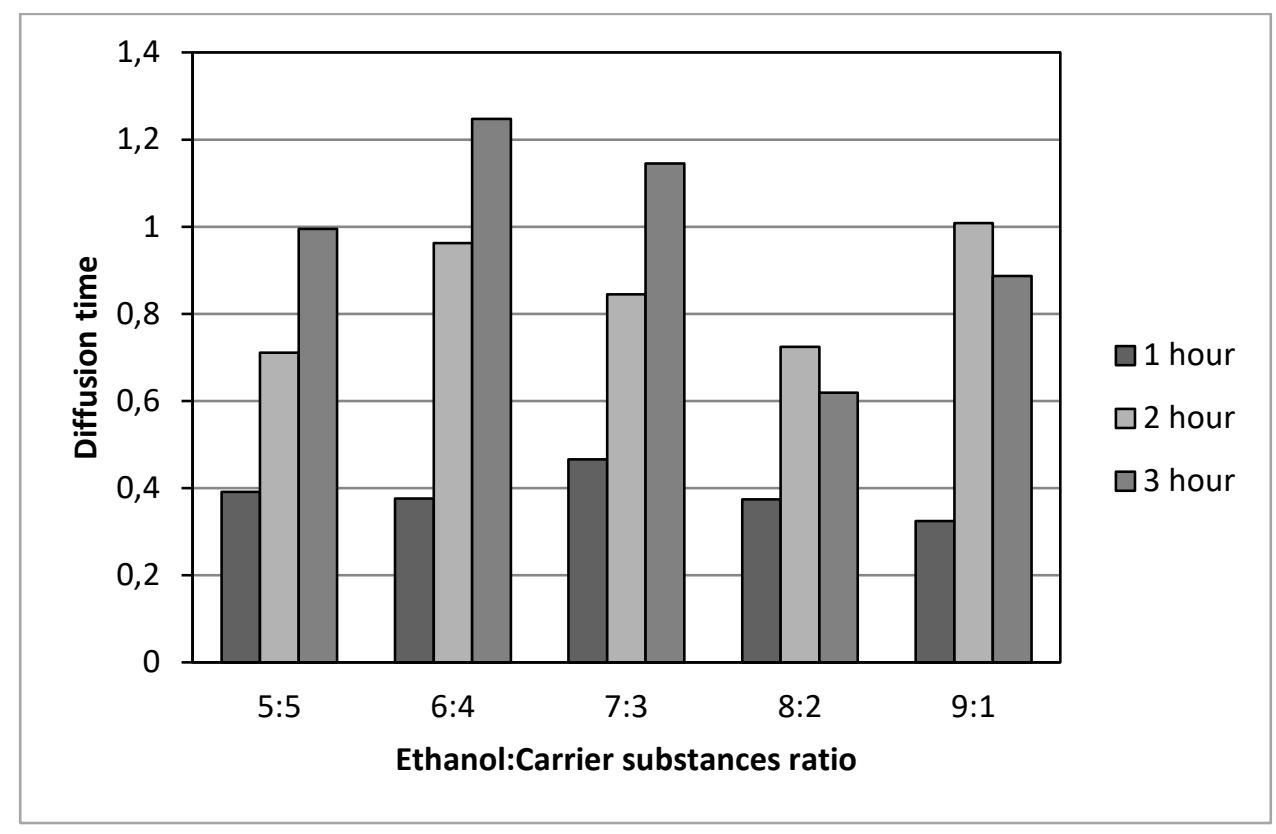

Fig. 3. The influence of the Ethanol: carrier substances ratio on the base of the diffusion solution.

\subsection{The effect of emulsifier on diffusion solutions}

Experimental results show that when using emulsifiers such as Tween 20, Tween 80, transparent solutions. In terms of homogeneity, both emulsifier samples used clearly observed no delamination of essential oils in the product matrix when using emulsifiers. The influence of emulsifiers on product volatility is shown in Figure 4. Survey results show that emulsifier Tween 80 has more volatility than Tween 20 . Therefore, Tween 80 is selected. as the main emulsifier in the solution for the next study. The influence of Tween 80 content on the background of the survey solution sample is shown in Figure 5. Through the survey results, it was found that the surfactant Tween 80 accounted for $3 \%$ of the obtained solution with high stability, less viscous. The reason is that the amount of surfactant is just enough to 
break down the emulsion particles to the micrometer size, when increasing the emulsifier content from 5-9\%, a large amount of emulsifier makes the diffusion solution become viscous and difficult to use. use. In contrast, when using $1 \%$ low emulsion content leads to the unstable system, layer separation phenomenon, so use $3 \%$ content to investigate the next factor.

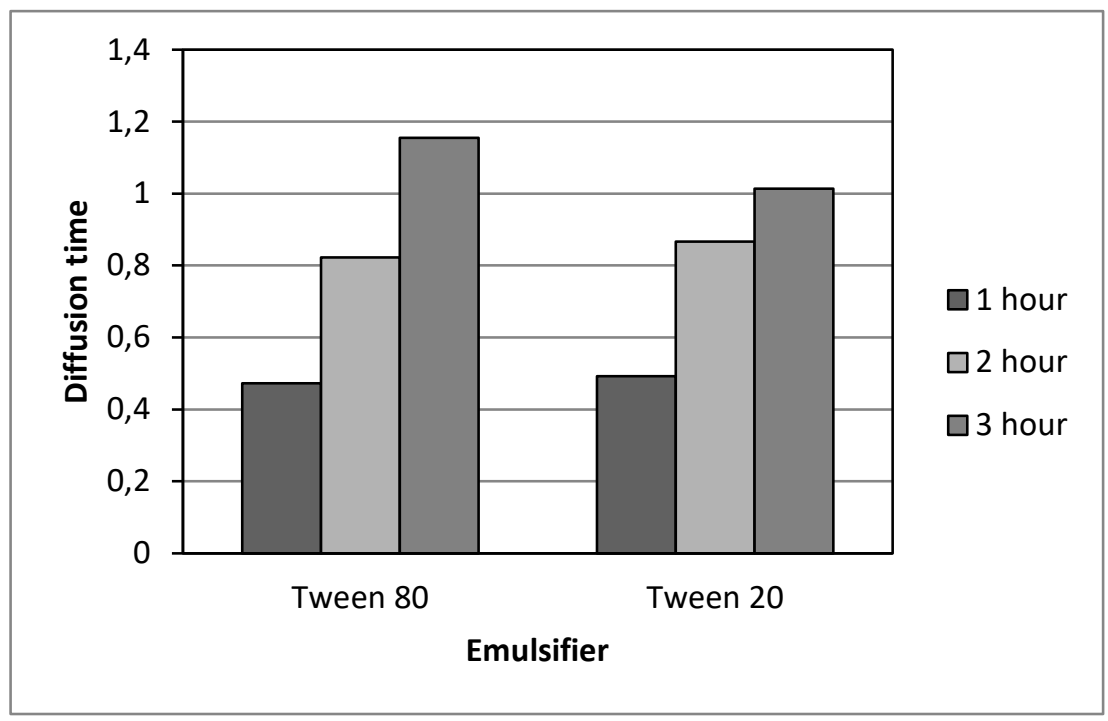

Fig. 4. The influence of the emulsifier on the base of the diffusion solution.

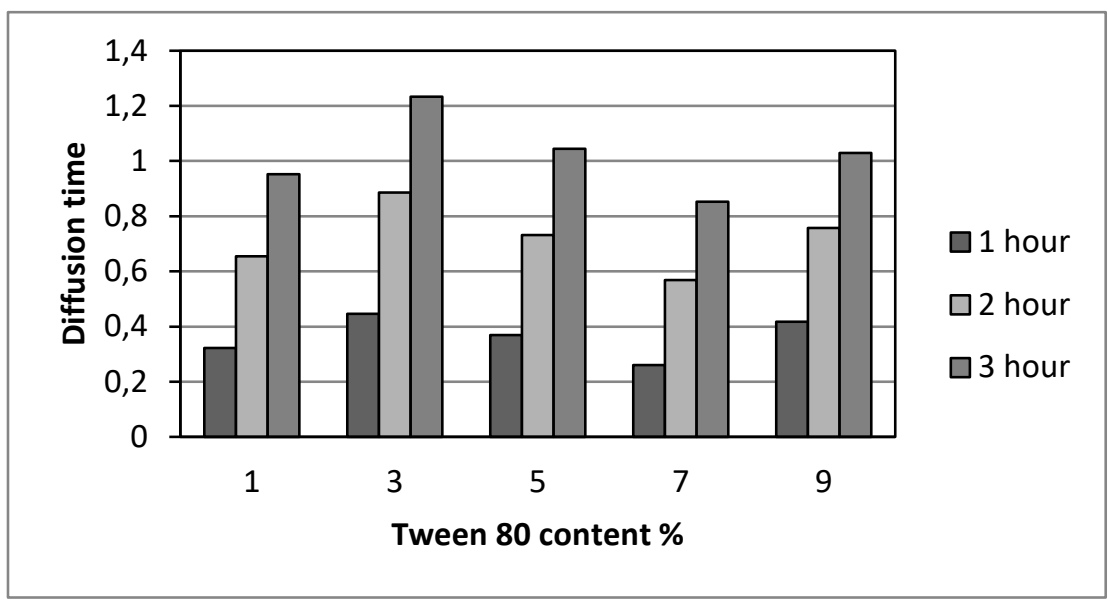

Fig. 5. The influence of the Tween 80 content on the base of the diffusion solution.

\subsection{The impact of storage conditions on the diffusion solution}

Figure 6 shows the influence of storage conditions (room temperature, acceleration, thermal shock) on the diffusivity of the solution. The solution samples when stored under three conditions did not have much difference in the sample diffusivity. On the other hand, samples stored under storage conditions showed no organoleptic changes compared with samples under normal conditions. Limonene, responsible for the basic sensory properties of pomelo essential oils, has been identified as the most prominent compound in pomelo peel essential 
oils accounting for $95 \%$, along with other terpenes. Experimental studies have demonstrated that D - limonene forms allergenic oxidation products, such as limonene oxide, limonene hydroperoxide, and R - (-) - carvone during handling and storage. The hydroperoxide of Dlimonene is unstable and readily decomposes into secondary oxidation products. Therefore, the product preservation process also affects the quality of the product and harms the health of consumers $[19,20]$.

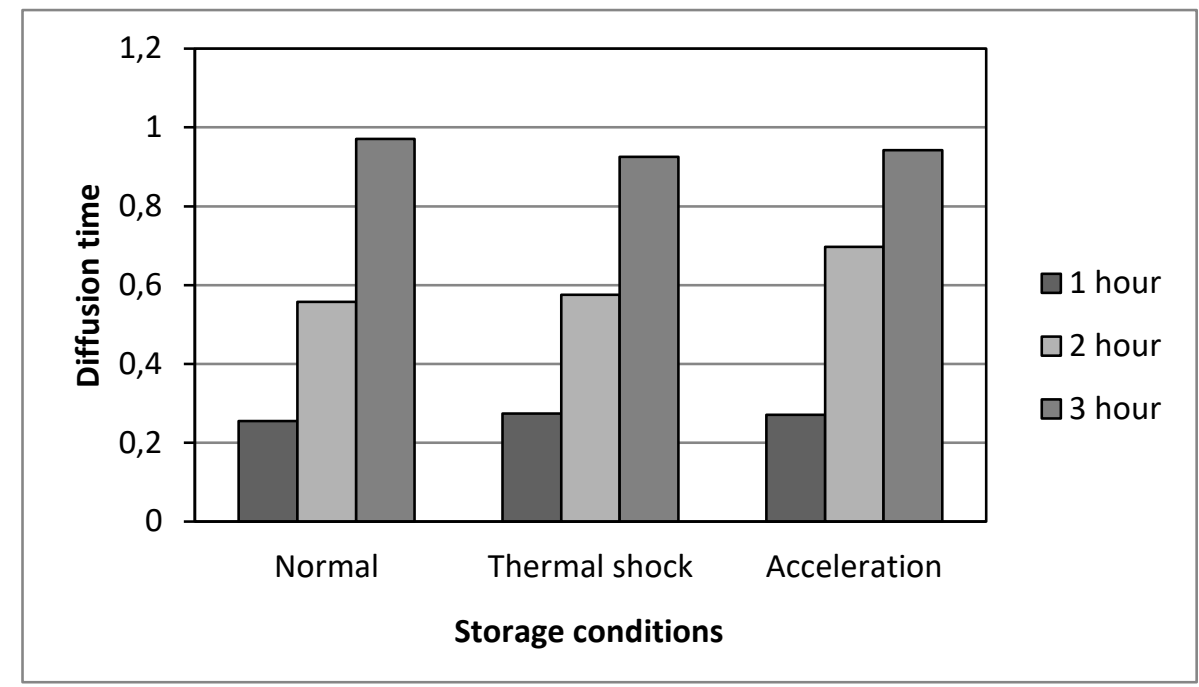

Fig. 6. The effect of storage conditions on the diffusion solution

\section{Conclusions}

Through the process of experimental research, with the application potentials and diverse activities of pomelo essential oil, D-limonen as a main ingredient is the main scent-creating agent to help effectively deodorize. The study has given a diffusion formula containing $4 \%$ pomelo essential oil content, 3\% emulsifier and a 6:4 solvent:carrier ratio, which exhibits good stability and evaporation efficiency compared to other formula. Although pomelo essential oil has diverse potential, it is important to be aware of unwanted harmful properties because it is a mixture of multi-active compounds that are easily denatured through the influence of ambient conditions. From the results of research to build production technology processes, perfect product formulas, assess consumer senses and evaluate according to prescribed standards. On that basis, the study evaluates the ability to commercialize products and successfully transfer production technology to enterprises to produce and develop products for the consumer market.

Acknowledgments. This study was funded by project no. 50/2020 NTMN.TW, 05/08/2020 (Ministry of Science and Technology of Vietnam).

\section{References}

1. T.T.K. Ngan, T.T. Hien, P.H. Danh, L.T.H. Nhan, L.X. Tien, IOP Conf. Ser. Mater. Sci. Eng. 959 (2020)

2. M. Dreger, K. Wielgus, Herba Pol. 59 (2014)

3. J. Veerasophon, P. Sripalakit, A. Saraphanchotiwitthaya, J. Adv. Pharm. Technol. Res. 
$11(2020)$

4. P.J. Jadhav, A.N. Mane, S.S. Gilda, V.B. Kumbhar, M.B. Jadhav, A.A. Ghadge, Al. Jadhav, World J. Pharm. Res. World J. Pharm. Res., 6 (2016)

5. T.K.N. Tran, T.H. Tran, T.H.N. Le, T.L. Xuan, N.Q.A. Phan, T.I. Cam, G.B. Long, IOP Conf. Ser. Mater. Sci. Eng., 736 (2020)

6. T.T.K. Ngan, T.T. Hien, X.T. Le, T.T. Anh, P.M. Quan, M.H. Cang, T.T. Le Ngoc, V.T. Danh, L.N.Y. Trung, T.Q. Toan, Asian J. Chem. 31 (2019)

7. T.T.K. Ngan, N.C. Huong, X.T. Le, P.Q. Long, T.Q. Toan, D.M.H. Vo, V.T. Danh, L.N.Y. Trung, T.A. Trieu, Asian J. Chem. 31 (2019)

8. T.H. Tran, L.K. Ha, D.C. Nguyen, T.P. Dao, L.T.H. Nhan, D.H. Nguyen, T.D. Nguyen, D.-V.N. Vo, Q.T. Tran, L.G. Bach, Processes 7, 56 (2019)

9. T. Tran, H. Nguyen, D. Nguyen, T. Nguyen, H. Tan, L. Nhan, D. Nguyen, L. Tran, S. Do, T. Nguyen, Processes 6, 206 (2018)

10. T.T.K. Ngan, D.T.T. Thuy, T.T. Tuyen, C.T. Inh, H.T. Bich, P.Q. Long, N.Q. Chien, H.T. Kieu Linh, L.N. Yen Trung, N.Q. Tung, D.C. Nguyen, L.G. Bach, T.Q. Toan, Asian J. Chem., 32 (2019)

11. T.H. Tran, P.T.N. Nguyen, T.N. Pham, D.C. Nguyen, T.P. Dao, T.D. Nguyen, D.H. Nguyen, D.V.N. Vo, X.T. Le, N.T.H. Le, L.G. Bach, IOP Conf. Ser. Mater. Sci. Eng., 479 (2019)

12. T.T.K. Ngan, N. van Muoi, P.M. Quan, M.H. Cang, Asian J. Chem. 32 (2020)

13. B. Uysal, F. Sozmen, O. Aktas, B.S. Oksal, E.O. Kose, Int. J. Food Sci. Technol., 46 (2011)

14. F.G. Kirbaşlar, I. Boz, J. Essent. Oil Res., 18 (2006)

15. D.A. Prasad, B.R. Prasad, D.K. Prasad, P. Shetty, K.N.S. Kumar, J. Appl. Pharm. Sci. 6 (2016)

16. Z. Xie, Q. Liu, Z. Liang, M. Zhao, X. Yu, D. Yang, X. Xu, J. Anal. Methods Chem., 8 (2013)

17. W.O. Okunowo, O. Oyedeji, L.O. Afolabi, E. Matanmi, Am. J. Plant Sci. 4 (2013)

18. J.S. Baik, S.S. Kim, J.A. Lee, T.H. Oh, J.Y. Kim, N.H. Lee, C.G. Hyun, J. Microbiol. Biotechnol., 18 (2008)

19. J. Sun, Altern. Med. Rev., 12, 3 (2007)

20. M. Matura, A. Goossens, O. Bordalo, B. Garcia-Bravo, K. Magnusson, K. Wrangsjö, A.T. Karlberg, J. Am. Acad. Dermatol., 47 (2002) 